\title{
La comunicación entre gabinetes y periodistas a través de la web 2.0: el caso de meneXtra.com
}

\author{
María José CantalaPiedRa GonZÁlez \\ Universidad del País Vasco/Euskal Herriko Unibertsitatea \\ mariajose.cantalapiedra@ehu.es \\ Leire ITURREGUI MARDARAS \\ Universidad del País Vasco/Euskal Herriko Unibertsitatea \\ leire.iturregui@ehu.es \\ Daniel García GonZÁLEZ \\ Universidad del País Vasco/Euskal Herriko Unibertsitatea \\ daniel.garcia@ehu.es
}

Recibido: $31 / 07 / 2012$

Aceptado: 30/10/2012

\begin{abstract}
Sumario
Los gabinetes de comunicación han visto ampliarse la potencialidad de las herramientas que utilizan, pero gran parte de sus tareas continúan fiándose a métodos tradicionales de contacto. En el marco de un proyecto de investigación financiado por el Gobierno Vasco ${ }^{1}$, este trabajo propone un estudio del entorno digital en las relaciones entre gabinetes y periodistas. Entre sus conclusiones destaca que sí ha florecido una ingente cantidad de plataformas para la distribución de notas de prensa, por ejemplo, pero que prácticamente son inexistentes las vías digitales de convocatorias de eventos, determinantes en las agendas de estos profesionales y que carecen además de foros en los que compartirlos. El equipo investigador ha concebido y diseñado una posible estructura digital para suplir esa carencia: menextra.com.
\end{abstract}

Palabras clave: gabinetes de comunicación, comunicación institucional, convocatorias de prensa, web 2.0.

\section{Communication between cabinets and journalists through the web 2.0: the case of meneXtra.com}

\begin{abstract}
The communication offices have seen extending the potentiality of tools used in their day to day work, nevertheless, a large part of their most common tasks keep on relying on traditional contact methods as the fax or the ordinary mail. In the frame of a research project funded by the Basque Government, this work proposes a study of the current digital environment of the relations between PRs and journalists. It emphasizes that there has bloomed an enormous quantity of distribution platforms of press releases in the network, but that there is no digital routes of call of events, so determinant in the agendas of the journalists. This paper proposes an approach to the original conception and design of a digital structure that could serve in the future to fill this gap; meneXtra.com.
\end{abstract}

Keywords: communication offices, institutional communication, call of press events, 2.0 web.

\section{Referencia normalizada}

CANTALAPIEDRA GONZÁLEZ, María José; ITURREGUI MARDARAS, Leire y GARCÍA GONZÁLEZ, Daniel (2012): "La comunicación entre gabinetes y periodistas a través de la web 2.0: el caso de meneXtra.com". Estudios sobre el mensaje periodístico. Vol. 18, núm. especial octubre, págs.: 213222. Madrid, Servicio de Publicaciones de la Universidad Complutense.

${ }^{1}$ Proyecto Saiotek de la convocatoria 2011, financiado por el Departamento de Industria, Innovación, Comercio y Turismo del Gobierno Vasco y dirigido por María José Cantalapiedra González, Directora del Departamento de Periodismo II de la Facultad de CCSS y de la Comunicación de la UPV/EHU 
Sumario: 1. Introducción. 2. Metodología. 3. Desarrollo; 3.1. Gabinetes y periodistas: hacia una herramienta bidireccional; 3.2. meneXtra.com.4. Conclusiones. 5. Referencias bibliográficas

\section{Introducción}

Desde la aparición de los primeros gabinetes de comunicación sus rutinas de trabajo y su relación con los periodistas han sufrido notables cambios derivados, principalmente, de la implantación y desarrollo de las nuevas tecnologías. En este proceso, se ha señalado especialmente a internet como elemento transformador central: "Internet sirve, incluso, de instrumento modulador de rutinas profesionales, lenguajes, procedimientos, protocolos, códigos de conducta, métodos de planeamiento y gestión" (Hoyo, 2006:81). Las notas de prensa, comunicados, ruedas de prensa, declaraciones, dossier de prensa, entrevistas, filtraciones o encuentros informales son algunas de las principales herramientas que los gabinetes de comunicación utilizan en su relación con periodistas (Canel, 2007) para captar su atención y lograr un espacio en los medios. Pero en los últimos años, además, las páginas web se han consolidado como herramienta clave en la imagen y comunicación corporativa (Losada Díaz, 2009; Sánchez y Rodríguez, 2010) y han incorporado un nuevo recurso al servicio de los gabinetes de comunicación: las Salas de Prensa Virtuales o SPV.

Se ha definido la sala de prensa virtual como "una serie de páginas dentro del sitio web corporativo que ofrece los servicios tradicionales del gabinete de comunicación pero ahora a través de la web" (Sánchez y Rodríguez, 2010: 5-6). Se ha alabado su utilidad y hay incluso quien afirma que "se han convertido en uno de los principales instrumentos de relación entre organizaciones y medios de comunicación" (Castillo y Almansa, 2005: 134). Si bien la Asociación de Directivos de Comunicación en España señalaba en su Anuario de 2012 que "hasta hace poco, los correos electrónicos de los periodistas se veían inundados de convocatorias, notas de prensa, fotos e información de multitud de empresas" internet ha inspirado la creación de nuevos instrumentos para las Relaciones Públicas, "entre ellas la más destacada es la sala de prensa 2.0, un lugar que facilita el acceso de los medios a información relevante de la compañía" (DirCom, 2012: 68).

En el ámbito académico se han llevado a cabo en España varias investigaciones centradas en las SPV de empresas, instituciones y asociaciones que analizan los elementos de los que disponen y carecen, los espacios 2.0 que albergan, los contenidos audiovisuales, su actualización o su desarrollo tecnológico (García Orosa y Capón García, 2005; González-Herrero y Ruiz de Valbuena, 2006; Castillo, 2006; Carrillo y Parejo, 2009; Sánchez y Rodríguez, 2010; Ruiz Mora et al., 2010). El objeto de investigación de los estudios varía - webs de Universidades, empresas, instituciones o asociaciones del tercer sector - pero todos ellos comparten diagnóstico: la mayoría de SPVs analizadas responde a lo que García Orosa (2009:25) califica de "gabinete on line 1.2", que realiza un "volcado literal de los instrumentos y documentos que el gabinete tradicional utiliza".

Ciertamente, en los últimos años se ha llevado a cabo un importante desarrollo, cuantitativo y cualitativo, de las SPV y resulta evidente que facilitan el acceso a la información de una empresa o institución, de modo que los periodistas "pueden acudir a la Web en cualquier momento y nutrirse de un material muy útil para su trabajo dia- 
rio" (Carrillo y Parejo, 2009). Sin embargo, García Orosa afirma que los profesionales de los medios no utilizan "de forma periódica y sistematizada" las SPV, principalmente, debido a su escasa actualización (García Orosa, 2009:44). Es decir, aún prima la función de las Salas de Prensa Virtuales como repositorio de material periodístico frente a su papel como herramienta al servicio de la interacción entre gabinetes y periodistas.

El principal objetivo del gabinete de comunicación reside en atraer a los periodistas para lograr un espacio en los medios. Para ello, resulta fundamental adecuarse a sus necesidades, ser conscientes de la saturación de información, emails y alertas que reciben, conocer los ritmos de las redacciones y tratar de encajar en la agenda de los medios. "Todos los actos noticiosos deben ser convocados con la suficiente anterioridad para que los medios de comunicación puedan prever la inclusión o no del tema entre los que conformarán el noticiario de ese día" (Álvarez y Caballero, 1997:181). Pero el margen de acción es breve, no más allá de dos o tres días, porque tal y como apuntan Arroyo y Yas (2003: 39), "el trabajo periodístico no permite demasiada planificación. Los redactores saben lo que harán hoy y mañana, pero muy probablemente ignoran lo que estarán haciendo dentro de tres días". Estos autores insisten, además, en la dificultad de lograr el "control de una variable que, por definición, nunca podemos controlar del todo: la competencia entre las organizaciones por aparecer en los medios de comunicación" (Arroyo y Yas, 2003: 39). Precisamente, en la actualidad no existe una herramienta que ponga en contacto las agendas de estos dos grandes grupos de profesionales. Por ello, en el marco de un proyecto de investigación financiado por el Gobierno Vasco, se ha tratado de diseñar una estructura digital que supla esta carencia: meneXtra.com.

\section{Metodología}

Para desarrollar la investigación que se presenta se ha creado un equipo de trabajo formado por periodistas con experiencia en medios y en comunicación institucional o empresarial, expertos en nuevas tecnologías y diseño. Tras un primer análisis de la bibliografía existente, se ha llevado a cabo un estudio de las SPV de instituciones y empresas de la CAPV así como de las herramientas digitales disponibles en la web para facilitar la relación entre periodistas y gabinetes. Todo ello ha permitido elaborar un diagnóstico inicial sobre las salas de prensa virtuales así como el objetivo y funcionalidad de las plataformas existentes. Se ha constatado la ausencia de una herramienta que ponga en común las agendas de ambas partes a fin de facilitar el trabajo de cada una de ellas y la comunicación entre las mismas.

En una segunda fase se han llevado a cabo varias sesiones de trabajo para identificar las necesidades de ambas partes, periodistas y responsables de empresas o instituciones, en su interacción diaria. Para ello, se ha diseñado una metodología centrada en la técnica de simulación (Sodupe et al., 2008; Wolfe y Crookall, 1998) en los que cada profesor ha interpretado distintos roles y desarrollado acciones de comunicación como responsables de gabinetes de instituciones y empresas y como periodistas. En este proceso se han identificado las necesidades de profesionales de medios - qué informaciones necesitan y cuáles no, en qué tiempos, en qué formato, a través de qué 
canales-y los deberes y necesidades de los responsables de gabinetes - qué condiciona la planificación de un evento, qué información deben trasladar de manera prioritaria, cómo incorporar nuevas herramientas a sus rutinas de trabajo sin que su uso suponga un trabajo añadido sino un instrumento a su servicio -.

A partir de la información recogida se elaboró un listado de procedimientos de trabajo y necesidades de ambas partes, así como las tareas de las fuentes emisoras, en este caso, los gabinetes de comunicación. Durante este proceso resultó vital identificar los elementos prescindibles, ya que el objetivo principal residía en obtener una plataforma digital que se limitara a los elementos esenciales para esa interacción. La última fase del trabajo consistió en la transformación de los conceptos manejados en diseños reales para una estructura digital que pudiera servir de comunicación entre ambos grupos de profesionales.

\section{Desarrollo}

En la revisión de las herramientas que instituciones de la Comunidad Autónoma del País Vasco (CAPV) y empresas vascas con presencia en el IBEX 35 han habilitado en internet para su relación con los medios (Cantalapiedra et al., 2012). Llama la atención, en este sentido, la diferencia entre empresas e instituciones en el uso de las TIC en su relación con los medios de comunicación. Las primeras han desarrollado una mayor inversión y esfuerzo en sistemas de recogida de información en sus webs corporativas. En las instituciones se percibe una apuesta por el desarrollo de tecnologías para alojar en sus webs información de interés para los medios, pero la publicación de materiales dirigidos a periodistas es escasa y pocas veces está actualizada.

El ámbito institucional y el tamaño que representa resulta, a su vez, determinante para el impulso de instrumentos al servicio de la comunicación. Si a nivel autonómico existen plataformas como Irekia que acogen las ruedas de prensa ofrecidas por miembros del equipo de gobierno, audios, fotos, notas de prensa e, incluso, informaciones montadas con videos editados, a nivel provincial destaca únicamente la Diputación Foral de Bizkaia, con una SPV ordenada, dotada de un sistema de alertas y convocatorias vía mail y SMS, y que incluye notas de prensa, ruedas de prensa, imágenes y audios. Todo cronológicamente ordenado y con un motor de búsqueda por temática. Por su parte, las Diputaciones Forales de Araba y Gipuzkoa carecen de SPVs avanzadas. La alavesa sí habilita una sección de prensa y de actualidad en su home con notas de prensa, fotos, agenda y clipping; la guipuzcoana dispone únicamente de un apartado de actualidad con noticias, una TV corporativa con conferencias y videos, pero sin materiales dirigidos a medios de comunicación. Respecto a las webs municipales, en ellas apenas existen en las webs municipales salas de prensa virtuales o secciones dirigidas a medios de comunicación, ni siquiera en las tres capitales. La mayoría acoge secciones de noticias y agenda, muchas veces con informaciones que se publican en formato pdf o poco actualizadas, pero no cuentan con salas de prensa ni secciones específicas dirigidas a los medios con materiales propios.

Por otro lado, se ha constatado la creación de una ingente cantidad de plataformas de distribución de información que ofrecen la posibilidad de enviar y distribuir notas de prensa. Existen disponibles en la red recursos para publicación de estas informa- 
ciones y agregadores de noticias institucionales, tanto en el ámbito internacional, webwire.com, prweb.com o mmd.com- como en el estatal -notasdeprensa.eu o sunotadeprensa.com-. Esta última, para la que es imprescindible suscribirse, está diseñada con el apoyo de la Asociación de la Prensa de Sevilla y se presenta en la web de la asociación como "sitio web innovador que actúa como Gabinete de Prensa Online y permite la publicación y distribución de notas de prensa, comunicados, convocatoria de medios, ruedas de prensa"2.

Además, algunos servicios van más allá de la mera distribución, al ofrecer un seguimiento de la repercusión de la información enviada a medios a través de un listado de periodistas que han consultado la noticia y de los principales medios que la han publicado -mmd.com- o una compilación de informaciones sobre temas específicos para su redistribución a nivel, incluso, europeo -como press4transport.eu, centrado en el transporte terrestre en la UE-. Se ha desarrollado una importante cantidad de programas, así mismo, para la organización de eventos -gotomeeting.com, eventprosoftware.com-, algunas de ellas de pago. Ninguna web establece filtros geográficos sobre sus usuarios, de manera que se ofrece un mismo contenido para quienes la visitan desde Bilbao, Madrid o Londres.

$\mathrm{Al}$ igual que ocurría con las SPV, la mayoría de las plataformas diseñadas para el apoyo de los gabinetes de prensa en su labor diaria se limita a la función de distribución y acumulación sin aportar interacción entre gabinetes y periodistas.

\subsection{Gabinetes $y$ periodistas: hacia una herramienta bidireccional}

El objetivo de un gabinete de comunicación es captar la atención de los medios, para lo que resulta fundamental conocer la agenda de estos: contra qué otras informaciones compiten por el mismo espacio temporal, temático y territorial. En la actualidad no existe una herramienta que ponga en contacto las agendas de estos dos grandes grupos de profesionales. El equipo investigador del proyecto propone, por ello, la creación de un entorno digital que pudiera servir como punto de encuentro de las agendas de unos y otros.

Las convocatorias de prensa son herramientas centrales en la comunicación entre medios e instituciones para la construcción de la agenda. Mediante una convocatoria se anuncia la celebración de un evento - una rueda de prensa, un desayuno informativo o unas declaraciones $-\mathrm{y}$ se invita a periodistas a acudir al mismo. Además de servir de introducción de un tema en los informativos del día, pretende hacerse un hueco en su agenda. Se trata, por tanto, del instrumento que en mayor medida condiciona la agenda de los medios, puesto que exige la disposición de recursos humanos durante un tiempo determinado así como su traslado al lugar en el que se desarrolle el acto.

Los medios de comunicación reciben diariamente decenas de convocatorias para asistir a eventos, muchos de ellos solapados en horarios. La decisión, por tanto, de convocar un acto si existe margen para su planificación -cuando no se trata de una in-

2 Disponible en la web de la Asociación de la Prensa de Sevilla: http://www.asociacionprensa.org/es/inicio/noticias-censo-de-expertas/271-nace-sunotadeprensacom-primer-gabinete-de-comunicacion-online.html 
formación de última hora-, debería estar condicionada por las previsiones que los medios tienen para ese día. Sin embargo, los convocantes, en su mayoría responsables de gabinetes de comunicación, carecen de acceso a esa información: las SPV de webs institucionales y corporativas apenas publican convocatorias (Cantalapiedra et al., 2012) y las plataformas digitales diseñadas para el apoyo del trabajo en los gabinetes se centran en la distribución de las mismas a los medios pero no en su difusión pública y coordinada. Únicamente las agencias de prensa publican las previsiones del día, a primera hora, y una vez los actos han sido convocados.

Las convocatorias constituyen, a su vez, una de las herramientas al servicio de los gabinetes de comunicación que menos se ha adaptado a las nuevas tecnologías. Si bien las TIC pueden ayudar al gabinete de prensa por ofrecer, precisamente, "un canal ágil para la convocatoria de ruedas de prensa, así como para el envío de material" (Canel, 2007:271), únicamente se ha aprovechado el potencial del correo electrónico o del envío de SMS para el primero de esos fines, descuidando de manera prácticamente absoluta las oportunidades que la web 2.0 brinda en este ámbito. Aún es habitual el uso del fax para hacer llegar las convocatorias o las llamadas de teléfono para confirmar el evento y la asistencia al mismo. La investigación que aquí se presenta propone la siguiente alternativa para ello.

\section{2. meneXtra.com}

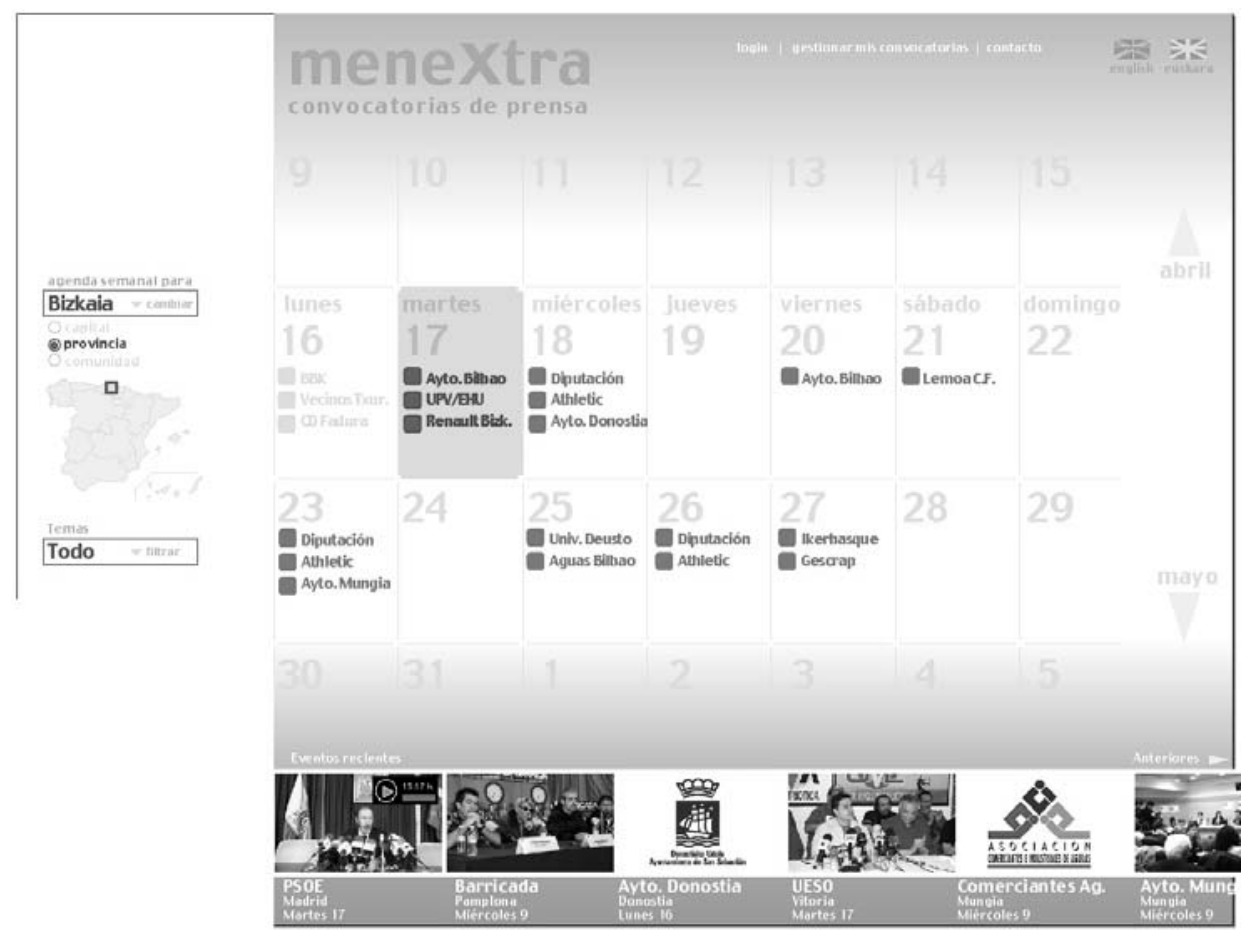

Figura 1: Aspecto general del interfaz de meneXtra.com 
La plataforma web resultante de la investigación responde a una estructura web al servicio de la comunicación entre responsables de gabinetes y periodistas que podría contribuir al avance hacia una relación 2.0 en la comunicación institucional y corporativa, favoreciendo la interacción entre ambos gremios e incorporando a los gabinetes una herramienta - bajo la denominación propuesta de meneXtra.com - que propicie su transformación hacia el gabinete de comunicación online.

A través de esta plataforma las fuentes tendrían acceso a información de la que antes sólo disponían los medios. Su diseño responde a un formato de agenda y su objetivo se centra en acoger de manera ordenada las convocatorias de eventos, atendiendo a su temática, territorialidad y temporalidad. Por tanto, meneXtra.com trata de satisfacer tanto las necesidades de medios de comunicación -temático y territorial-, como las de los gabinetes-temático y temporal-.

La plataforma ofrecería un acceso abierto e intuitivo, con un diseño orientado a usuarios con perfil periodístico y sin necesidad de suscripción. Por el contrario, sí es imprescindible inscribirse para publicar eventos: la persona responsable del gabinete deberá incluir sus datos personales y se llevará a cabo un proceso de verificación de los mismos. Una vez finalizado este trámite, el acceso será siempre personalizado para ese usuario, autorizado además para gestionar en la web la comunicación y actividades de más de una entidad.

Los responsables de los gabinetes pueden anunciar qué material estará disponible tras el acto, en qué formato y si el evento será retransmitido en directo vía streaming o a su finalización. Permite programar la publicación de convocatorias y gestionar varias simultáneamente - pasadas y futuras- en borradores, visibles únicamente para el gabinete organizador.

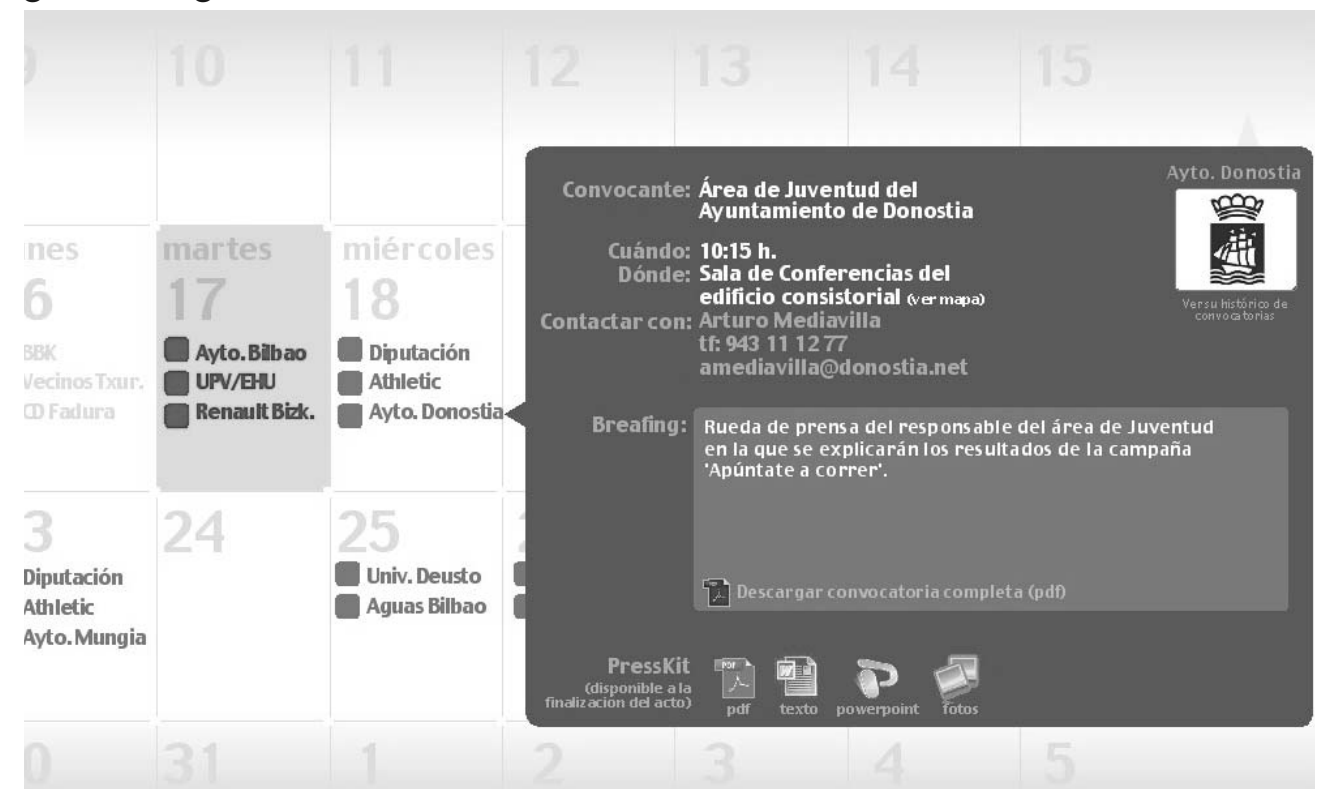

Figura 2: Vista de un evento convocado en meneXtra.com 
Una herramienta de estas características permitía a instituciones o empresas de menor tamaño, miembros de la comunidad activa en la comunicación institucional, publicar sus informaciones en igualdad de condiciones que aquellas entidades con más recursos. Además, de permitir el seguimiento del trabajo desarrollado por responsables de gabinetes con mayor protagonismo, aquellos otros gabinetes más modestos podrán compartir agenda y tendrían a su alcance una potente herramienta de generación de contenidos audiovisuales o materiales elaborados.

\section{Conclusiones}

Las herramientas creadas para favorecer la comunicación entre periodistas y gabinetes -SPVs, distribuidores de notas de prensa, etc.-, se limitan a una función de almacenaje de información o envío de mensajes preparados sin facilitar la interacción entre gabinetes y periodistas. Su utilización supone, por otro lado, una duplicación de esfuerzos por parte de los profesionales de las RRPP, que además de desarrollar sus labores tradicionales deben dotar de contenido y gestionar estas nuevas herramientas.

Entre las plataformas diseñadas destaca la falta de atención prestada a la agenda de los medios. En la actualidad sólo es posible conocer las previsiones informativas del día a primera hora de la jornada, a través de las agencias de prensa y una vez los actos han sido convocados. Las convocatorias de prensa son, en este sentido, las grandes olvidadas en este ámbito de comunicación en la web 2.0. La investigación que se presenta pretende suplir esa carencia.

Se constata la necesidad de una estructura digital centrada en la publicación de convocatorias que permite conocer la agenda informativa a medios y gabinetes, así como la interacción entre los mismos, para lo que el equipo investigador ha creado una plataforma bajo el nombre de meneXtra.com. El diseño de la nueva herramienta nace del conocimiento de las prácticas profesionales, adaptando la informática a las necesidades comunicativas $-\mathrm{y}$ no a la inversa- $\mathrm{y}$ con el reto de acoger únicamente lo esencial para una relación interactiva y efectiva entre ambos gremios.

A través de meneXtra.com se propone una herramienta de uso intuitivo para profesionales de la comunicación que busca facilitar el trabajo de los gabinetes en su interacción con periodistas. Además de simplificar la elaboración, publicación y envío de las convocatorias, pretende servir de agenda informativa conjunta - compartida por medios y gabinetes $-\mathrm{y}$ favorecer la coordinación de eventos informativos y pondría a disposición de las entidades más modestas un instrumento de gran potencial para publicar sus eventos online, con recursos audiovisuales y junto a aquellas de mayor tradición comunicativa.

\section{Referencias Bibliográficas}

ÁLVAREZ, Tomás y CABALLERO, Mercedes (1997): Vendedores de imagen. Los retos de los nuevos gabinetes de comunicación. Barcelona, Paidós Papeles de comunicación 18.

ARROYO, Luis y YAS, Magali (2003): Los cien errores de la comunicación de las organizaciones. Madrid, ESIC. 
CANEL, María José (2007): Comunicación de las instituciones públicas. Madrid, Tecnos.

CANTALAPIEDRA, María José; ITURREGUI, Leire y GARCÍA, Daniel (2012): "Una aproximación a la comunicación entre gabinetes y periodistas", en CALDEVILLA GONZÁLEZ, David (coord.): Aplicaciones del EEES a partir de la web 2.0 y 3.0. Madrid, Visión Libros, pp. 17-39.

CARRILLO DURÁN, María Victoria y PAREJO, Macarena (2009): "Las salas de prensa virtuales de los gabinetes de comunicación de las universidades públicas españolas", en IV Congreso de la CiberSociedad. Crisis analógica, futuro digital. http://www.cibersociedad.net/congres2009/ [Consultado el 28 de agosto de 2012].

CASTILLO ESPARCIA, Antonio (2006): "Public Relations and Press Room. Análisis de las salas de prensa virtuales de las grandes empresas de España", en Razón y Palabra. $\mathrm{n}^{\circ} 49$, http://www.razonypalabra.org.mx/anteriores/n49/mesa7.html [Consultado el 15 de julio de 2012]

CASTILLO ESPARCIA, Antonio; ALMANSA MARTÍNEZ, Ana (2005): "Relaciones públicas y Tecnología de la Comunicación. Analisys de los sitios de prensa virtuales". Organicom. n³. pp. 133-149.

DirCom, Asociación de Directivos de Comunicación (2012): Anuario de la Comunicación 2012. Madrid, Enlaze3 Prinmt Management.

GARCÍA OROSA, Berta (2009): Gabinetes de comunicación on line. Claves para generar información corporativa en la red. Sevilla, Comunicación Social.

GARCÍA OROSA, Berta y CAPÓN GARCÍA, José Luis (2005): “Gabinets on line y redes sociales virtuales”, en LÓPEZ GARCÍA, Guillermo (coord.): El ecosistema digital: modelos de comunicación, nuevos medios y público en Internet. Valencia, Universitat de València, pp. 197-228.

GONZÁLEZ-HERRERO, Alfonso y RUIZ DE VALBUENA, Miguel (2012): "Trends in online media relations: Web-based corporate press romos in leading international companies". Public Relations Review n³2. pp. 267-275.

HOYO, Javier del (2008): "Nuevas redes y empresa. Tecnologías web y su aplicación a la comunicación corporativa". Telos $\mathrm{n}^{\mathrm{0}} 66$, pp. 79-82.

LOSADA DÍAZ, José Carlos (2009): "Los 10 frentes de la comunicación corporativa”. Doxa Comunicación nº. pp. 145-164.

RUIZ MORA, Isabel María; SALAR OLMEDO, Silvia y ÁLVAREZ NOBELL, Alejandro (2010): "Salas de prensa virtual, redes sociales y blogs: posibilidades de la comunicación 2.0", en V Congreso Internacional de Investigación en Relaciones Públicas, Barcelona, 13 y 14 de mayo de 2010.

SÁNCHEZ PITA, Fernando y RODRÍGUEZ GORDO, Carlos (2010): “Tendencias en la construcción de salas de prensa virtuales de las principales empresas del índice bursátil IBEX 35", en II Congreso Internacional de Comunicación 3.0. Universidad de Salamanca. 4 y 5 de octubre de 2010. http://campus.usal.es/ comunicacion3punto0/comunicaciones/050.pdf [Consultado el 28 de agosto de 2012] . 
SODUPE, Kepa; MOURE, Leire e ITURRE, Maite (2009): La simulación como recurso pedagógico en Relaciones Internacionales: la implementación del Modelo de Naciones Unidas UPV-EHU (EHUMUN). Leioa, Universidad del País Vasco.

\section{María José CANTALAPIEDRA GONZÁLEZ}

mariajose.cantalapiedra@ehu.es

Profesora Titular del Departamento de Periodismo II

Facultad de Ciencias Sociales y de la Comunicación.

Universidad del País Vasco - Euskal Herriko Unibertsitatea (UPV/EHU)

\section{Leire ITURREGUI MARDARAS}

leire.iturregui@ehu.es

Profesora Laboral Interina del Departamento de Periodismo II

Facultad de Ciencias Sociales y de la Comunicación

Universidad del País Vasco - Euskal Herriko Unibertsitatea (UPV/EHU)

\section{Daniel GARCÍA GONZÁLEZ}

daniel.garcia@ehu.es

Profesor Asociado del Departamento de Periodismo II

Facultad de Ciencias Sociales y de la Comunicación

Universidad del País Vasco - Euskal Herriko Unibertsitatea (UPV/EHU)

Departamento de Periodismo II

Facultad de Ciencias Sociales y de la Comunicación

Universidad del País Vasco UPV/EHU

Barrio Sarriena

48940 Leioa 